\title{
Minkowski Circle Packings on the Sphere
}

\section{Fejes Tóth}

Mathematical Institute, Hungarian Academy of Sciences, Pf 127, H-1364 Budapest, Hungary

To the fiftieth birthday of my son Gábor

\begin{abstract}
We consider $n$ caps on the sphere such that none of them contains in its interior the center of another. We give an upper bound for the total area of the caps, which is sharp for $n=3,4,6$, and 12 and is asymptotically sharp for great values of $n$.
\end{abstract}

\section{Introduction, Results}

A set of balls is said to be a Minkowski set if none of its elements contains in its interior the center of another. Replacing in a Minkowski set of balls each ball with a concentric ball of radius half as big as the original, we obtain a packing of balls which we call a Minkowski packing. Minkowski sets, Minkowski packings of balls, and their variants have been investigated because of their connection with the geometry of numbers and the theory of functions [1], [2] as well as because of their intrinsic interest [3]-[9]. This paper deals with Minkowski sets and Minkowski packings of circles (spherical caps) on the sphere.

In Section 2 we prove two theorems.

Theorem 1. The density of a Minkowski packing of $n>2$ circles on the sphere is at most

$$
\frac{n}{2}\left(1-\frac{1}{2} \sin ^{-1} \omega_{n}\right)
$$

where

$$
\omega_{n}=\frac{n}{n-2} \frac{\pi}{6}
$$

Theorem 2. The density of a Minkowski set of $n>2$ circles on the sphere is at most

$$
n\left(1-\frac{1}{4} \sin ^{-2} \omega_{n}\right) \text {. }
$$


Both bounds are sharp for $n=3,4,6$, and 12, and asymptotically sharp for great values of $n$.

Section 3 contains some open problems and remarks.

\section{Proof of Theorems 1 and 2}

Throughout this section we operate on the unit sphere. We use the same symbol for a domain and its area.

Let $c_{1}, \ldots, c_{n}$ be circles with radii $r_{1}, \ldots, r_{n}$ and centers $O_{1}, \ldots, O_{n}$, which form a Minkowski packing. We start the proof of Theorem 1 with the case of three circles. We may suppose that $r_{1}>\pi / 3$. Then $O_{2}$ and $O_{3}$ lie in a circle of radius $\pi-2 r_{1}$ which implies that $r_{2}<\pi-2 r_{1}$ and $r_{3}<\pi-2 r_{1}$. It follows that

$$
\frac{c_{1}+c_{2}+c_{3}}{4 \pi}<\frac{1}{2}\left(3-\cos r_{1}+2 \cos 2 r_{1}\right)
$$

which is less than the density bound 0.75 obtained from (1) for $n=3$.

Now we show that it is enough to prove Theorem 1 for $n>3$ circles such that $\max \left(r_{1}, \ldots, r_{n}\right) \leq 58.4^{\circ}$. Suppose that $r_{1}=58.4^{\circ}$ and that $O_{1}$ is the north pole. Then the centers $O_{2}, \ldots, O_{n}$ lie in a circle $\Gamma$ of radius $R=180^{\circ}-2 \times 58.4^{\circ}=63.2^{\circ}$ with center at the south pole. Let $T_{k}$ be the maximum total area of $k$ circles which along with $c_{1}$ constitute a Minkowski packing. It is not difficult to show that, for $k=3,4$, and 5 , $T_{k}$ consists of $k$ congruent circles whose radius $\varrho_{k}=\arcsin (\sin (\pi / k) \sin R)$ is half of the side length of a regular $k$-gon inscribed in $\Gamma . T_{6}$ is attained by six circles of radius $R / 2=31.6^{\circ}$. (Note that $R / 2$ is just a little bit smaller than $\varrho_{5} \approx 31.64$.) We list the approximate values of $T_{k}$ :

$$
\begin{array}{ccccc}
k & 3 & 4 & 5 & 6 \\
T_{k} & 6.8913 & 5.6383 & 4.6710 & 5.5898
\end{array}
$$

Obviously, the biggest radius of the circles yielding $T_{k}$ for $k>6$ is less than $R / 2$. Thus, for $k>6, T_{k}$ is certainly smaller than

$$
2 \pi\left(1-\cos \frac{3 R}{2}\right) \approx 6.8089
$$

showing that for $k \geq 3$ we have $T_{k} \leq T_{3}$. Therefore the density of the packing is at most

$$
\frac{2 \pi\left(1-\cos 58.4^{\circ}\right)+T_{3}}{4 \pi} \approx 0.7864 \text {. }
$$

This is smaller than the bound $0.845 \ldots$ obtained from (1) for $n=4$ and hence, by the monotonity of (1), smaller than the bound obtained for any $n \geq 4$. In the case of $r_{1}>58.4^{\circ}$, similar considerations yield even smaller density bounds.

Let $c$ and $c^{\prime}$ be two disjoint circles with radii $r$ and $r^{\prime}$ and centers $O$ and $O^{\prime}$, respectively. Let $\sigma$ be the (spherical) segment $O O^{\prime}$. Let $M$ be the midpoint of the segment with endpoints $\sigma \cap \mathrm{bd} c$ and $\sigma \cap \mathrm{bd} c^{\prime}$. Let $l$ be the line (great circle) drawn through $M$ 
orthogonally to $\sigma$. We call $l$ the midline of $c$ and $c^{\prime}$. We say that a point lies nearer to $c$ than to $c^{\prime}$ if it lies on the same side of $l$ as $c$.

Let $c$ be a circle of the packing. Let $C$ be the cell of $c$ defined as the set of points which are nearer to $c$ than to any other circle of the packing.

Let $c^{\prime}$ be another circle of the packing which does not contain the point diametrically opposite to $O$. Let $\Delta$ be the triangle bounded by the line $O O^{\prime}$, the midline of $c$ and $c^{\prime}$, and a tangent to $c^{\prime}$ passing through $O$. Let $s$ be the sector of $c$ lying in $\Delta$, and let $\omega$ be its angle. In the case when $c^{\prime}$ touches $c$, we write $\Delta^{*}, s^{*}$, and $\omega^{*}$ for $\Delta, s$, and $\omega$. We show that

$$
\frac{c}{C} \leq \frac{s^{*}}{\Delta^{*}}
$$

where $C$ is the cell of $c$.

First we prove that

$$
\frac{s}{\Delta} \leq \frac{s^{*}}{\Delta^{*}}
$$

Let $t$ be the distance between $O$ and $O^{\prime}$, and let $d=\frac{1}{2}\left(t+r-r^{\prime}\right)$ be the distance of $O$ from the midline of $c$ and $c^{\prime}$. Let $D$ denote the digon defined as the convex hull of $O, c^{\prime}$, and the point opposite to $O$. Consider the array of circles inscribed in $D$. In this array, $d$ is an increasing function of $t$. Approaching $c^{\prime}$ toward $c, s$ remains constant but $\Delta$ decreases so that $s / \Delta$ increases. If $\omega \leq \omega^{*}$, we stop approaching $c^{\prime}$ at the moment when $t=2 r$. If, on the other hand, $\omega>\omega^{*}$, we approach $c^{\prime}$ toward $c$ until it touches $c$ and observe that in this situation $\omega / \Delta$ is a decreasing function of $\omega$. Therefore, replacing $\omega$ by $\omega^{*}, s / \Delta$ increases on.

For $t=2 r$ we have

$$
\frac{s}{\Delta}=\frac{\omega(1-\cos r)}{\omega-\arcsin (\cos d \sin \omega)}=F_{r}(\omega)
$$

where $d=\left(3 r-r^{\prime}\right) / 2$ and $\sin r^{\prime}=\sin \omega \sin 2 r$. A computer investigation of $F_{r}(\omega)$ and its derivative — kindly performed by J. Linhart (Salzburg) — has shown that, for $r \in$ $\left(0,58.4^{\circ}\right), F_{r}(\omega)$ is, for $0<\omega \leq \omega^{*}$, strictly increasing, confirming (4). (For $r=$ $58.4^{\circ}$, the maximum of $F_{r}(\omega)$ is attained at $\omega \approx 73.009^{\circ}$, which is less than $\omega^{*}=$ $\arcsin (\sin r / \sin 2 r) \approx 73.125^{\circ}$.)

Turning to the proof of (3), let $c_{1}$ and $c_{2}$ be two circles corresponding to two consecutive sides of $C$. Let $A$ be the angular region bounded by the half-lines $O O_{1}$ and $O O_{2}$. We claim that

$$
\frac{A \cap c}{A \cap C} \leq \frac{s^{*}}{\Delta^{*}}
$$

Assuming that $\overline{O O_{1}}<\overline{O O_{2}}$, we rotate $c_{2}$ about $O_{1}$ so that $\overline{O O_{2}}$ decreases until $\overline{O O_{2}}=$ $\overline{O O_{1}}$. In any position of $c_{2}$ let $S$ be the set of points which are nearer to $c$ than either to $c_{1}$ or to $c_{2}$. Let the initial position of $c_{2}$ be such that $O O_{2}$ touches $c_{1}$. Now $A \cap c / A \cap S \leq s^{*} / \Delta^{*}$ with equality only if $c_{1}$ touches $c$. During the rotation of $c_{2}, A \cap c / A \cap S$ decreases until the intersection $I$ of the midlines of $c$ and $c_{1}$ and $c$ and $c_{2}$ lies outside of $A$. As soon as $I$ gets into $A, A \cap c / A \cap S$ increases. In the final position, when $\overline{O O_{2}}=\overline{O O_{1}}, c_{1}$, and 
$c_{2}$ are separated from each other by the line passing through $O$ and the midpoint of the segment $\mathrm{O}_{1} \mathrm{O}_{2}$, so that, in view of (4),

$$
\frac{A \cap c}{A \cap C} \leq \frac{A \cap c}{A \cap S} \leq \frac{s^{*}}{\Delta^{*}},
$$

as stated. Equality holds only if $c, c_{1}$ and $c_{2}$ mutually touch each other.

In what follows, we write $\omega^{*}=\omega$, keeping in mind that $\omega$ satisfies

$$
\sin \omega=\frac{1}{2 \cos r} \text {. }
$$

In the triangle $\Delta^{*}$, let $\varphi$ be the angle opposite to $r$. Then $\cos \varphi=\sin \omega \cos r=\frac{1}{2}$, so that $\varphi=\pi / 3$ and $\Delta^{*}=\omega-\pi / 6$. In view of (3), we have

$$
C \geq 2 \pi\left(1-\frac{\pi}{6 \omega}\right)=f(\omega)
$$

Consider $f(\omega)$ as a function $g(c)$ of

$$
c=2 \pi\left(1-\frac{1}{2} \sin ^{-1} \omega\right) .
$$

We have

$$
\frac{d g}{d c}=\frac{d g}{d \omega}: \frac{d c}{d \omega}=\frac{\pi}{3} \frac{\sin ^{2} \omega}{\omega^{2} \cos \omega}
$$

Writing $h(\omega)=\left(\sin ^{2} \omega\right) /\left(\omega^{2} \cos \omega\right)$, we have

$$
\begin{aligned}
\omega^{4} \cos ^{2} \omega h^{\prime}(\omega) & =\omega \sin \omega\left(2 \omega \cos ^{2} \omega+\omega \sin ^{2} \omega-\sin 2 \omega\right) \\
& =\omega \sin \omega\left(2 \omega-\sin 2 \omega-\omega \sin ^{2} \omega\right) \geq \omega \sin \omega\left(\frac{8 \omega^{3}}{6}-\omega \sin ^{2} \omega\right) \\
& =\omega^{2} \sin \omega\left(\frac{4}{3} \omega^{2}-\sin ^{2} \omega\right) \geq 0
\end{aligned}
$$

This implies that $g(c)$ is convex so that we can use Jensen's inequality:

$$
4 \pi \geq C_{1}+\cdots+C_{n} \geq n g(\bar{c})=n 2 \pi\left(1-\frac{\pi}{6 \omega}\right),
$$

where $\bar{c}$ is the average area of the circles $c_{1}, \ldots, c_{n}$ and $\omega$ is the pertaining value of it according to (7).

The last inequality yields

$$
\omega \leq \frac{n}{n-2} \frac{\pi}{6}=\omega_{n},
$$

whence

$$
\bar{c} \leq 2 \pi\left(1-\frac{1}{2} \sin ^{-1} \omega_{n}\right),
$$

completing the proof of Theorem 1. 
Next we turn to the proof of Theorem 2. For any circle $c$ of radius $r$, let $S(c)$ denote the area of the circle whose radius is $2 r$. Using the facts that $c=2 \pi(1-\cos r)$ and $S=2 \pi(1-\cos 2 r)$, we get

$$
\frac{d S}{d c}=\frac{d S}{d r}: \frac{d c}{d r}=4 \cos r
$$

which implies that $S(c)$ is concave. Thus, by Jensen's inequality, $\bar{S}$, the average area of the circles in the Minkowski set of circles associated with our Minkowski packing, satisfies $\bar{S} \leq S(\bar{c})$. In view of $(8), \bar{c} \leq 2 \pi(1-\cos r)$, where $\cos r=1 /\left(2 \sin \omega_{n}\right)$. Therefore,

$$
\bar{S} \leq 2 \pi(1-\cos 2 r)=4 \pi \sin ^{2} r=4 \pi\left(1-\frac{1}{4} \sin ^{-2} \omega_{n}\right),
$$

as asserted in Theorem 2 .

\section{Concluding Remarks}

In the special case when all circles are congruent, Theorem 1 has been proved independently from each other by Hadwiger and the author. This is the earliest result in the vast literature about packing the sphere with equal circles.

In the Euclidean plane, the problems of the densest Minkowski circle packing and the densest Minkowski circle arrangement are equivalent. The densest Minkowski circle packing consists of equal circles, and the densest Minkowski circle arrangement arises from the densest packing of equal circles by replacing each circle by a concentric one twice as big as the original. The situation is similar on the sphere in the case of $3,4,6$, and 12 circles but quite different for 5 circles.

Let $c$ be a circle with center $O$ and radius $r \geq 45^{\circ}$. Let $c^{\prime}$ be a circle centered at a distance $2 r$ from $O$ whose tangents through $O$ make a right angle. The radius of $c^{\prime}$ is given by $\sin r^{\prime}=(\sin 2 r) / \sqrt{2}$. Four circles congruent to $c^{\prime}$ along with $c$ form a Minkowski packing with density $a(r)=\frac{1}{2}\left(5-\cos r-4 \cos r^{\prime}\right)$. On the other hand, the density of the associated Minkowski arrangement is $b(r)=\frac{1}{2}\left(5-\cos 2 r-4 \cos 2 r^{\prime}\right)$. It is easy to see that the maximum of $a(r)$ and $b(r)$ yield the density of the densest Minkowski packing and the density of the densest Minkowski arrangement of five circles. However, $a(r)$ and $b(r)$ attain their maxima at different values of $r$, namely, at $r \sim 48.899^{\circ}$ and $r \sim 48.590^{\circ}$.

Are there other values of $n \neq 5$, for which the problem of the densest Minkowski packing of $n$ circles is not equivalent with the problem of the densest Minkowski arrangement of $n$ circles?

In the Euclidean plane we define a Minkowski arrangement of centrally symmetrical convex disks by the requirement that no disk contains in its interior the center of another disk. Several properties of such arrangement are known [3], [4]. Special attention is due to Minkowski arrangements of homothetic disks. Reducing in such an arrangement each disk by a similitude about its center in the ratio $1: 2$ we obtain a Minkowski packing. It is very probable that the density of a Minkowski packing of centrally symmetric homothetic convex disks never exceeds the density of the densest lattice packing of one of the disks. 
We sketch a line of attack to the problem. Let the disks $c_{1}, c_{2}, \ldots$ with centers $O_{1}, O_{2}, \ldots$ form a Minkowski packing. We assume that the disks are smooth. Let $c_{i}$ and $c_{j}$ be two of them, let $s$ be the segment $O_{i} O_{j}$, and let $M$ be the midpoint of the segment joining $s \cap \operatorname{bd} c_{i}$ and $s \cap \operatorname{bd} c_{j}$. We draw a line $l$ through $M$ parallel to the tangents of $c_{i}$ and $c_{j}$ at the points $s \cap \mathrm{bd} c_{i}$ and $s \cap \mathrm{bd} c_{j}$. We say that a point $P$ is nearer to $c_{i}$ than to $c_{j}$ if $P$ and $c_{i}$ lie on the same side of $l$. Let $C_{i}$ be the set of points which lie nearer to $c_{i}$ than to any other disks. Now the problem is to find the arrangement of the disks for which the area $C_{i}$ attains its minimum. The above conjecture would be proved if we could show that in this problem we can restrict ourselves to the case when $C_{i}$ is a hexagon.

\section{Acknowledgement}

I express my thanks to E. Makai for many helpful comments.

\section{References}

1. Bateman, P., Erdős, P., Geometrical extrema suggested by a lemma of Besicovitch, Amer. Math. Monthly 58, 306-314 (1951).

2. Besicovitch, A. S., A general form of the covering principle and relative differentiation of additive functions, Math. Proc. Cambridge Philos. Soc. 41, 103-110 (1945).

3. Bleicher, M. N., Osborn, J. M., Minkowskian distribution of convex discs, Acta Math. Acad. Sci. Hungar. 18, 5-17 (1967).

4. Fejes Tóth, L., Minkowskian distribution of discs, Proc. Amer. Math. Soc. 10, 999-1004 (1965).

5. Fejes Tóth, L., Packings and coverings in the plane, Proc. Colloquium on Convexity (Copenhagen, 1965), Kobenhavns Univ. Mat. Inst., 1967, pp. 78-87.

6. Fejes Tóth, L., Minkowskian circle-aggregates, Math. Ann. 171, 97-103 (1967).

7. Florian, A., Zur Geometrie der Kreislagerungen, Acta Math. Acad. Sci. Hungar. 18, 341-358 (1967).

8. Molnár, J., Aggregati di cerchi di Minkowski, Ann. Mat. Pura Appl. 71, 101-107 (1966).

9. Reifenberg, E. F., A problem on circles, Math. Gaz. 32, 290-292 (1948).

Received September 11, 1997, and in revised form March 2, 1998. 